\title{
Women's Heart Health: Differences in Heart Disease in Women Compared to Heart Disease in Men
}

Vicki L. Zeigler ${ }^{*}$

A s I sat around the table at the annual Go Red for Women ${ }^{\text {ти” }}$ luncheon in Denton, Texas, United States in February, I was struck by this year's theme "Hearts Around the World." Throughout my career in pediatric cardiovascular nursing in the last 40 years, I never even considered the fact that heart disease would ever surpass breast cancer (or any other forms of cancer combined) as the number one killer of women in the United States; the statistics from other countries are even more staggering. For example, one in eight women in the United Kingdom die from heart disease annually and almost 500000 women in the United Kingdom have had a previous myocardial infarction (MI) or heart attack. This editorial will highlight the differences in heart disease in women compared to heart disease in men. Although risk factors for cardiovascular disease are incredibly important, the discussion of them is beyond the scope of this editorial. The importance of heart disease in women has been recently highlighted by two Scientific Statements from the American Heart Association $(1,2)$, both of which provide a dearth of information about the large body of evidence that has been gathered thus far regarding women's heart health.

First, heart disease is no longer a "man's" disease. Research has shown that not only are there differences among the sexes, but also among genders, ethnicities, and age groups. The definitions of sex and gender have been previously described and include genetic/biological differences in men and women and various perceived social conceptualizations among men and women, respectively. The outward physical appearance of men and women is generally easily discernable; however, the differences in cardiovascular anatomy, physiology, and effects of reproductive hormones are less obvious. For example, anatomic dimensions are smaller in women than men, which would logically lead one to believe that a blockage in a smaller vessel would cause more problems sooner rather than later, than in a larger one. A nearly perfect analogy to this is the concrete barriers and lane dimension decreases in construction zones. It is much harder to "squeeze" one's vehicle through these lanes than if they were construction-free. Imagine if this were plaque in an already smaller vessel.

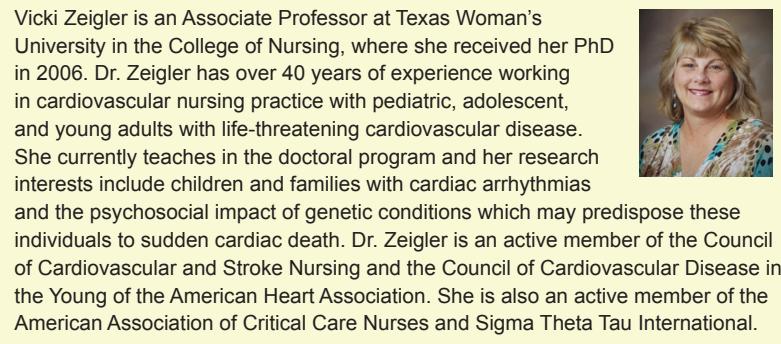

Other sex differences include the influence of hormones, various functions of the heart, situation-specific responses (such as stress and altitude), hematologic indexes, and electrocardiographic and electrophysiological indexes (2). One of the most common gender issues affecting women with suspected heart disease is the delay in seeking treatment when symptoms occur. This scenario was well illustrated in a video produced by a well know actress, Elizabeth Banks, entitled "Just a Little Heart Attack" (3). She uses humor to illustrate how many things women have to juggle every day to maintain their expected social roles. These roles include, but are not limited to, being a wife and mother, multitasking (doing laundry, feeding the cat, making breakfast and lunch for the kids), seeing her husband off to work, while simultaneously having the symptoms of a heart attack. Her phone is ringing in the background and she assures her son and husband that she is okay. She is not okay. Today's woman takes on many roles and some are even the "breadwinners" for their families. These gender issues, be all and do all, are becoming increasingly common in women and contribute to them ignoring the symptoms of the bodies they know so well. It is critical that we as healthcare providers, and by that I mean anyone involved in patient care, educate women to listen to their bodies. If the body is trying to tell them something, it usually means there is something wrong. Cultural differences and age differences in women are especially concerning. Women of color, particularly Black women, have a higher incidence of cardiovascular disease and commonly delay seeking treatment. In fact, the initial presenting symptom of coronary artery disease is often sudden cardiac death in Black women (2). Many of 
these differences are related to risk factors and cultural issues, but still require vigilance and increased attention by healthcare providers. The best thing that we can do as healthcare providers is to read and disseminate as much information as possible to our female patients as well as our communities about these issues related to women's heart health. Additionally, as we have seen in the occurrence of breast and ovarian cancers, cardiovascular disease is resulting in increased mortality over the past decade in younger and younger women (1).

Second, the symptoms once thought to be the "hallmark" of a heart attack are no longer the "typical" symptoms, especially in women. Specifically, the feeling of indigestion, the feeling of an elephant sitting on one's chest, and the sensation of pain radiating down the left arm are no longer the only symptoms in women. The seminal work of Dr. Jean McSweeney from Arkansas changed the landscape of how women specifically experience pain of a cardiovascular nature (2). Chest pain or discomfort remains a common symptom of a heart attack, but additional symptoms that may occur in women include pain or discomfort in one or both arms, the back, as well as the neck, jaw, or stomach. Shortness of breath with or without chest discomfort should not be ignored, yet neither should other symptoms such as nausea, light-headedness, or breaking out in a cold sweat. I urge you to take a moment and look at the video by Elizabeth Banks (3) and identify the symptoms that she was having during the video. She portrayed almost all of the ones presented here.

Third, one of the most common obstacles to survival of women experiencing symptoms of myocardial ischemia is the delay in seeking treatment and getting the necessary treatment. In addition to Blacks, Hispanics, and American Indians are particularly vulnerable to delays in seeking treatment and despite incredible improvements in the diagnosis and treatment of cardiovascular disease in women over the past 20 years, women are "less likely to be treated with guideline-directed medical therapies, less likely to undergo cardiac catheterization, and less likely to receive to receive timely reperfusion therapy" . This is appalling. Additionally, when women are treated for cardiovascular disease, they are more likely to remain hospitalized longer, have a higher incidence of dying while hospitalized, have more procedural/treatment complications, and have more readmissions than their male counterparts (1).

To say that cardiovascular disease in women is a public health priority is an understatement. There is absolutely no excuse for us to ignore this issue any longer. It is time for more research, proper diagnosis, timely and accurate treatment, and education. Again, as I sat at that table in February, I decided at that moment to increase by own advocacy and education of communities regarding women with cardiovascular disease. Why? Because the clock is ticking and it is time for a "wake up" call to action. We have learned so much over the past several decades, now it is time for us to use that knowledge to make a difference in the lives of women around the world.

\section{Ethical Issues}

Not applicable.

\section{Conflict of Interests}

The author has no conflicts of interest to disclose.

\section{References}

1. Mehta LS, Beckie TM, DeVon HA, et al. Acute myocardial infarction in women. A Scientific Statement from the American Heart Association. Circulation. 2016;133:916-947. doi: 10.1161/ CIR.0000000000000351.

2. McSweeney JC, Rosenfeld AG, Abel WM, et al. Preventing and experiencing ischemic heart disease as a woman: state of the science. A Scientific Statement from the American Heart Association. Circulation. 2016;133:1302-1331. doi:10.1161/ CIR.0000000000000.381

3. American Heart Association. Just a Little Heart Attack. American Heart Association Go Red for Women Editors. Dallas, TX. https://www.goredforwomen. org/about-heart-disease/symptoms_of_heart_ disease_in_women/just-a-little-heart-attack/.

Copyright (C) 2016 The Author(s); This is an open-access article distributed under the terms of the Creative Commons Attribution License (http://creativecommons.org/licenses/by/4.0), which permits unrestricted use, distribution, and reproduction in any medium, provided the original work is properly cited. 